\title{
Polarization Considerations for the Laser Interferometer Space Antenna
}

\author{
Eugene Waluschka ${ }^{a}$, Tracy R. Pedersen ${ }^{b}$, Paul McNamara ${ }^{c}$ \\ ${ }^{a}$ NASA/Goddard Space Flight Center/551.0, Greenbelt, MD 20771 \\ ${ }^{\mathrm{b}}$ Swales Aerospace, 5050 Powder Mill Rd, Beltsville, MD 20705 \\ ${ }^{c}$ University of Maryland, College Park, MD 20742
}

\begin{abstract}
A polarization ray trace model of the Laser Interferometer Space Antenna's (LISA) optical path is being created. The model will be able to assess the effects of various polarizing elements and the optical coatings on the required, very long path length, picometer level dynamic interferometry. The computational steps are described. This should eliminate any ambiguities associated with polarization ray tracing of interferometers and provide a basis for determining the computer model's limitations and serve as a clearly defined starting point for future work.
\end{abstract}

Keywords: LISA, gravitational wave, interferometer, polarization

\section{Introduction}

The Laser Interferometer Space Antenna (LISA) is to detect gravitational waves by means of three spacecraft (SC) in orbit about the sun using coherent laser links to measure changes in their relative distances. The laser wavelength is approximately 1.064 microns. Each SC will send to the other two a quasi-monochromatic Gaussian profile laser beam with a total outgoing power of about one Watt. Each received beam is attenuated as a result of diffraction and propagating 5 million kilometers. So that the captured beam's intensity is measured in tens of picowatts, it's wavelength is shifted due to the relative motion of the spacecraft and after reflection from a freely falling proof mass (or suitable equivalent) is detected, along with a superimposed local, also quasi-monochromatic, but off-set in frequency, laser beam as a beat note. At the main fringe counting detector, the "local" and received "far" beams are given by (1) and (2)

$$
\begin{array}{r}
\vec{E}_{l}(\vec{r}, t)=\left(\begin{array}{c}
A_{l}^{s}(\vec{r}, t) \cdot e^{i\left[\vec{k}_{f}(\vec{r}, t) \cdot \vec{r}-\omega_{f}(t): t+\phi_{l}^{t}(\vec{r}, t)\right]} \\
A_{l}^{p}(\vec{r}, t) \cdot e^{i\left[\vec{k}_{l}(\vec{r}, t) \cdot \vec{r}-\omega_{f}(t) \cdot t+\phi_{l}^{p}(\vec{r}, t)\right]}
\end{array}\right) \\
\vec{E}_{f}(\vec{r}, t)=\left(\begin{array}{c}
A_{f}^{s}(\vec{r}, t) \cdot e^{i\left[\vec{k}_{f}(\vec{r}, t) \cdot \vec{r}-\omega_{f}(t) \cdot t+\varphi_{f}^{s}(\vec{r}, t)\right]} \\
A_{f}^{p}(\vec{r}, t) \cdot e^{i\left[\vec{k}_{f}(\vec{r}, t) \cdot \vec{r}-\omega_{f}(t) t+\varphi_{f}^{p}(\vec{r}, t)\right]}
\end{array}\right)
\end{array}
$$

with $\vec{r}=(x, y, z)$ and $\vec{k}=\frac{2 \pi n}{\lambda} \hat{k}$. The intensity at any point on $\vec{r}$ on the detector is given by (3).

$$
I(\vec{r}, t) \sim\left|\vec{E}_{l}(\vec{r}, t)+\vec{E}_{f}(\vec{r}, t)\right|^{2}
$$

Upon substituting (1) and (2) into (3) we get an equation with the form of (4)

$$
I(\vec{r}, t) \sim A_{l}^{s} A_{f}^{s} \cos \left[\Delta \omega \cdot t+\Delta k+\Delta \varphi^{s}\right]+A_{l}^{p} A_{f}^{p} \cos \left[\Delta \omega \cdot t+\Delta k+\Delta \varphi^{p}\right] .
$$

The dependencies on $(\vec{r}, t)$ are still present, but not written. If we view the beat frequency (say about $1.0 \mathrm{Mhz}$ ) $f=\Delta \omega / 2 \pi$ as due to by the frequency off-set (neglecting laser frequency noise) of the lasers and the Doppler shift caused by the relative velocities of the spacecraft, then the gravitational wave signal is in the terms $\Delta \varphi^{s}$ and $\Delta \varphi^{p}$. Now, a time variation of any of the quantities in (4) is a departure from strictly monochromatic sinusoidal variation and is apt to produce 
frequency and phase shifts.

The intensity $I(\vec{r}, t)$ in (4) is at a point on the detector. The total signal (assuming uniform detector response) is given by (5) where the integration is over the area of the detector.

$$
I(t) \sim \iint_{a r e a} I(\vec{r}, t) d a
$$

LISA will detect a gravitational wave as a time varying (within the 0.1 to $0.0001 \mathrm{~Hz}$ range) strain, $\Delta L / L$. $\Delta L$ will be of the order of tens of picometers when $L$ is approximately 5 million kilometers, the distance between the LISA spacecraft. Noise sources which can obscure the gravitational signal, such as spacing changes between the primary and secondary mirrors of the telescope due to temperature changes, are routinely examined as part of an optical design analysis. Phase changes due to refractive index changes are also routinely examined as are optical coating thin film effects. However, phase changes due to electric field amplitude variations, $A_{l}^{s} A_{f}^{s}$ and $A_{l}^{p} A_{f}^{p}$ in (4), are typically small and usually not of concern. In the case of LISA the smallness of the gravitational wave signal requires a careful look at all possible noise sources, including phase changes due to electric field amplitude changes.

The following sections describe the current work, performed primarily at Goddard, in creating a LISA polarization ray trace (PRT) model which relies on existing commercial optical design and analysis computer codes and on in house analysis tools which are used to check and give insight into the computation. It should be noted that this is a work in progress. The immediate goal is to understand and string together the LISA specific PRT computational steps keeping in mind that the layout of the LISA optical bench and the design of the telescope are not final, but also works in progress. None-the-less having an end-to-end PRT model of LISA, at an early stage, gives insight into the capabilities and limitations of the readily available computational tools, such as ZEMAX and CODE-V, the polarization properties of a specific optical design and individual optical elements with their optical coatings.

\section{Varying Amplitude Produces Phase Shift}

A simple, if not totally rigorous, way of connecting the amplitude variation in (4) to the phase is to make use of the fact that the light incident on the LISA's photo-detector is strongly linearly polarized, hence one component of the incident electric field $A A^{s}(t) \gg A A^{p}(t)$. Then dividing (4) we get (6)

$$
\frac{I(\vec{r}, t)}{A_{l}^{s} A_{f}^{s}} \sim \cos \left[\Delta \omega \cdot t+\Delta k+\Delta \varphi^{s}\right]+\frac{A_{l}^{p} A_{f}^{p}}{A_{l}^{s} A_{f}^{s}} \cos \left[\Delta \omega \cdot t+\Delta k+\Delta \varphi^{p}\right] .
$$

As $\frac{A_{l}^{p} A_{f}^{p}}{A_{l}^{s} A_{f}^{s}} \cong 0$ hence $\cos \left(\frac{A_{l}^{p} A_{f}^{p}}{A_{l}^{s} A_{f}^{s}}\right) \cong 1$ and $\sin \left(\frac{A_{l}^{p} A_{f}^{p}}{A_{l}^{s} A_{f}^{s}}\right) \cong \frac{A_{l}^{p} A_{f}^{p}}{A_{l}^{s} A_{f}^{s}}$ we can write (7)

$$
\frac{I(\vec{r}, t)}{A_{l}^{s} A_{f}^{s}}-\cos \left(\frac{A_{l}^{p} A_{f}^{p}}{A_{l}^{s} A_{f}^{s}}\right) \cos \left[\Delta \omega \cdot t+\Delta k+\Delta \varphi^{s}\right]+\sin \left(\frac{A_{l}^{p} A_{f}^{p}}{A_{l}^{s} A_{f}^{s}}\right) \cos \left[\Delta \omega \cdot t+\Delta k+\Delta \varphi^{p}\right]
$$

and in the case of $\Delta \varphi^{p}=\Delta \varphi^{s}-\frac{\pi}{2}$ we have (8) and (9) giving an approximate magnitude to the effect.

$$
\begin{gathered}
\frac{I(\vec{r}, t)}{A_{l}^{s} A_{f}^{s}} \sim \cos \left(\frac{A_{l}^{p} A_{f}^{p}}{A_{l}^{s} A_{f}^{s}}\right) \cos \left[\Delta \omega \cdot t+\Delta k+\Delta \varphi^{s}\right]+\sin \left(\frac{A_{l}^{p} A_{f}^{p}}{A_{l}^{s} A_{f}^{s}}\right) \sin \left[\Delta \omega \cdot t+\Delta k+\Delta \varphi^{s}\right] \\
I(\vec{r}, t) \sim A_{l}^{s} A_{f}^{s} \cos \left[\Delta \omega \cdot t+\Delta k+\Delta \varphi^{s}-\frac{A_{l}^{p} A_{f}^{p}}{A_{l}^{s} A_{f}^{s}}\right] .
\end{gathered}
$$

When $\Delta \varphi^{p} \neq \Delta \varphi^{s}-\frac{\pi}{2}$ the connection between variation in amplitude and phase is a bit more involve. 


\section{Input, Output, Reference, and Acquisition Optical Paths}

The optical layout, as well as the PRT, are works in progress. A ray trace model of the current version is shown in fig. 1-2. Even though this layout is by no means final it is used for the usual optical analyses, such as sensitivity and diffraction, which lead up to a final design. It also provides a frame work with a sufficient number of optical elements which can be coated for a realistic PRT analysis. Although shown in Fig. 1, the acquisition path is not part of the polarization analysis.

Fig. 1 shows the "front-end" optics used in sending to and receiving laser light from the distant spacecraft. A closer view of some of the optics in the output/input path is shown in Fig.2.

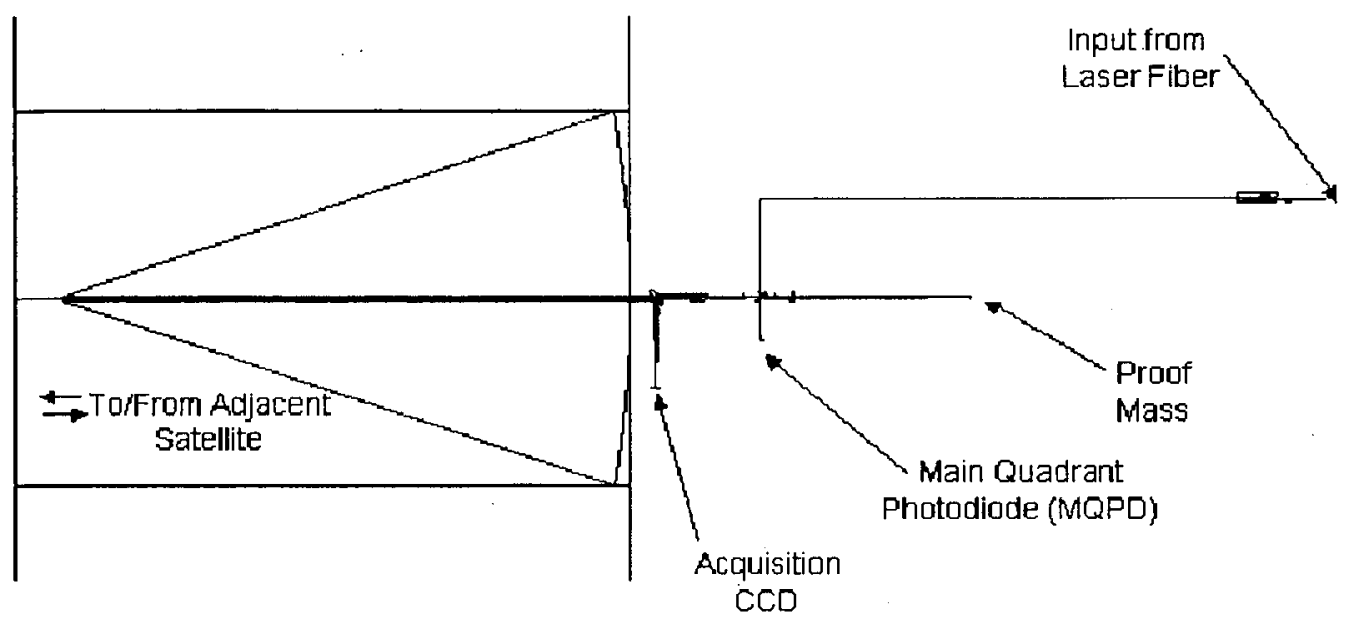

Fig. 1: Input, output, reference and acquisition paths.

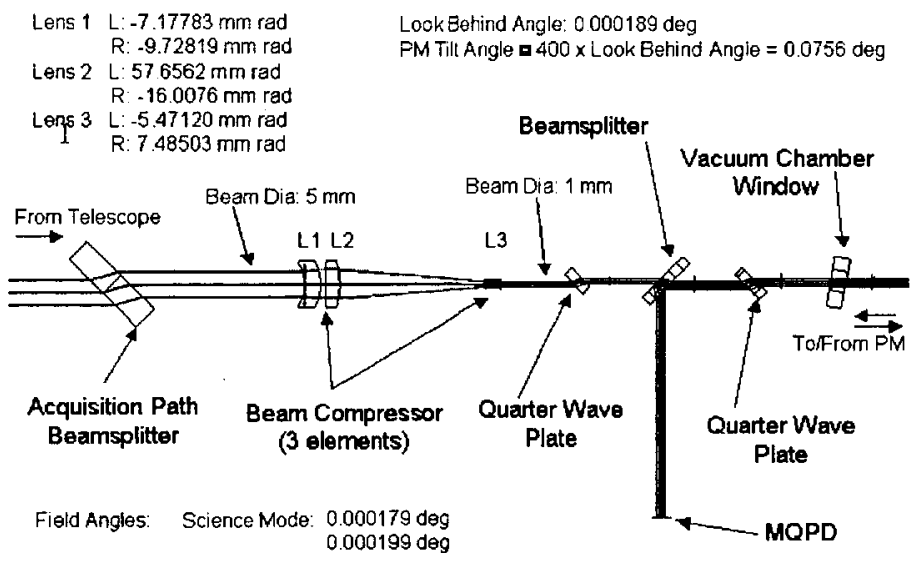

Fig. 2: Details of optical components 


\section{Polarization Ray Trace Modeling Approach}

The LISA PRT model is built up using commercial optical analysis software, such as CODE-V or ZEMAX and an in house FORTRAN program, RAYPKS. The advantage of using commercial software is that simply turning on the polarization switch allows one to perform a polarization ray trace. Default coatings are applied to surfaces and as a ray is traced the electric field, associated with that ray, is suitably modified by the surfaces. This approach is adequate for "standard optical" systems. However, for optical systems such as interferometers, which have multiple (dynamic) paths the use of off the shelf software is at times cumbersome. A fair amount of time is required to set up an optical multiple path configuration and the computed results are, at times, difficult to understand. This is especially true of the thin film "phase" associated with rays traversing different optical configurations and subsequently combined to produce a interference pattern. This is not to say that the commercial codes give incorrect results, just that it is difficult to confidently state that the computations are fully understood. The advantages of using a home-brewed code and having the source code is that all of the source code and hence computations are visible and extracting the desired information just requires a few "simple" code modifications (which need to be checked). The disadvantage of using a home-brewed code is the need to maintain both a ray trace code, a thin film code and the connection between them, also any "bugs" cannot be blamed on someone else.

\section{Laser to Telescope Aperture - Outgoing beam}

For the purposes of this discussion we assume that the light ray leaving the fiber in fig. 1 and going through the polarizer is strongly linearly polarized with the electric field perpendicular to the plane of the paper this being the " $\mathrm{S}$ " direction. The electric field, in a suitable coordinate frame attached to a ray, is given by the Jones vector (10)

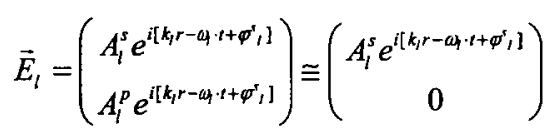

were we have made use of $A_{l}^{s} \gg A_{i}^{p} \cong 0$. Even though the discussion treats the electric field as a 2-dimensional vector the computation (in RAYPKS and undoubtedly in the commercial codes as well) treats it as a 3-dimensional vector. This approach avoids mostly conceptual difficulties associated with reflections.

This beam is reflected, in fig. 2, by a polarizing beamsplitter toward the telescope, but a small fraction of the beam does "leak" through the beam splitter and is used as the interferometric reference beam. The outgoing beam passes through a quarter wave plate having its crystal axis oriented to convert linear to circular polarized light (11).

$$
\vec{E}_{l} \cong e^{i\left[k_{l} r-a, t\right]}\left(\begin{array}{c}
A_{l}^{s} e^{i \varphi_{l}^{\prime}} \\
\left.A_{l}^{p} e^{i\left(\varphi^{p_{l}}+\frac{\pi}{4}\right)}\right)
\end{array}\right)
$$

Fig. 3 depicts the circular polarized light of the outgoing beam at the aperture of the telescope. (The ellipsoidal appearance of the circles in Fig. 3 is a plotting artifact.) The current LISA baseline design assumes circular polarized light between the $\mathrm{SC}$, however this may change.

Something to take note of is the fact that each ray, in a PRT, has a local coordinate frame attach with respect to which the electric field is specified. One axis of this coordinate system is parallel to the ray direction, another is parallel to the "S" and the other to the "P" directions, which are respectively normal and parallel to a plane of incidence. So that in general as a ray propagates from surface to surface the ray coordinate frame rotates. In our case the "S" and "P" directions, for the individual rays start off parallel, but by the time they reach the telescope exit aperture are not parallel as seen in fig. 4 . We need to take this into account when computing the far field at the entrance aperture of the distant telescope which has it's own local coordinate frame. 


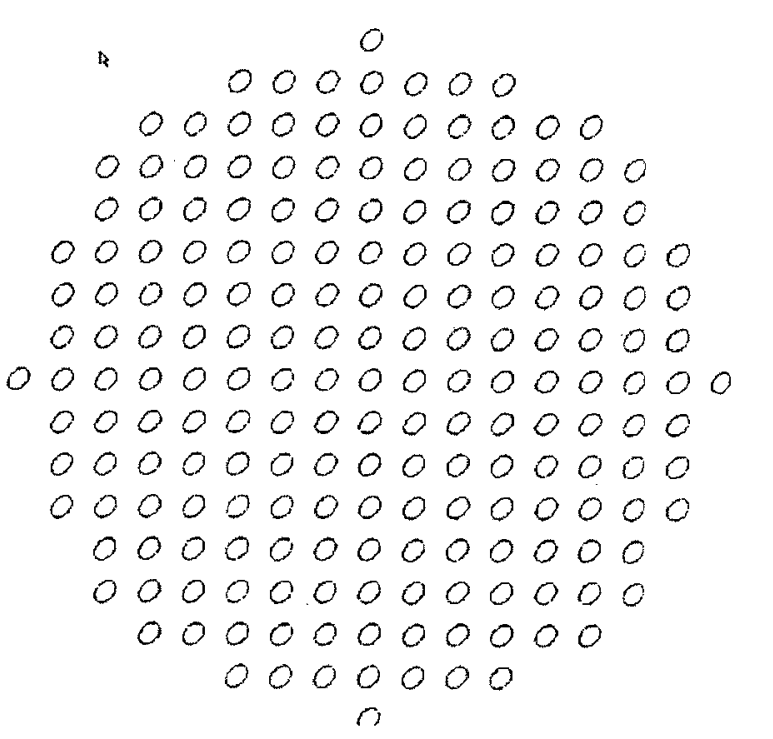

Fig.3: Circularly polarized light at the exit aperture of the telescope

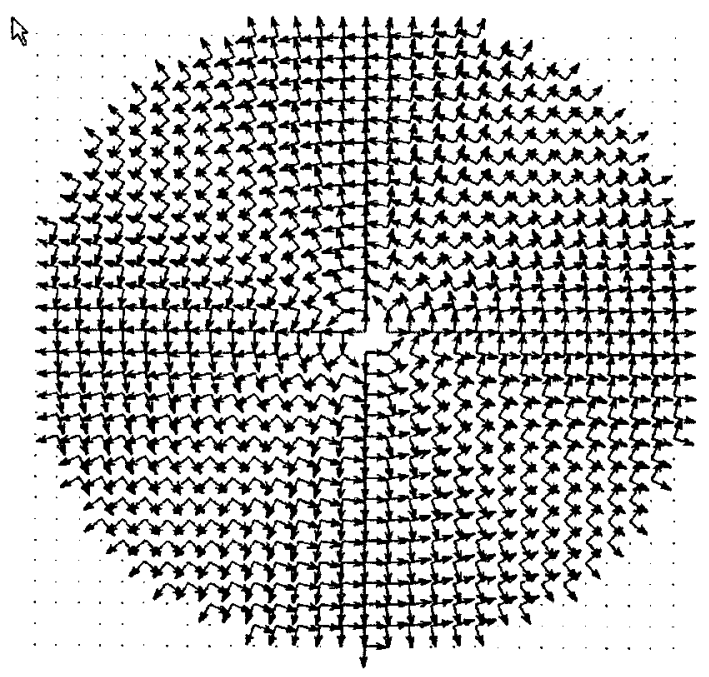

Fig 4: $\mathrm{S}$ and $\mathrm{P}$ directions for individual rays at aperture of telescope.

\section{Space Craft and Other Motions}

The motion of LISA's three SC about the sun, in the course of a year, is shown in fig. 5 where the center of the triangle is about one astronomical unit from the sun. Each SC is in its own suitably chosen orbit. In a locally inertial reference frame attached to one $\mathrm{SC}$ the motion of one of the other distant $\mathrm{SC}$ is shown in fig. 6 (generated by assuming Keplerian orbits of all three SC). Each SC rotates, with respect to its local inertial frame, in the course of a year. In this rotating frame, attached to the SC, the motions of the other two distance SC are relatively small and in the course of a year the angular separation varies by only about one degree, fig. 7 .

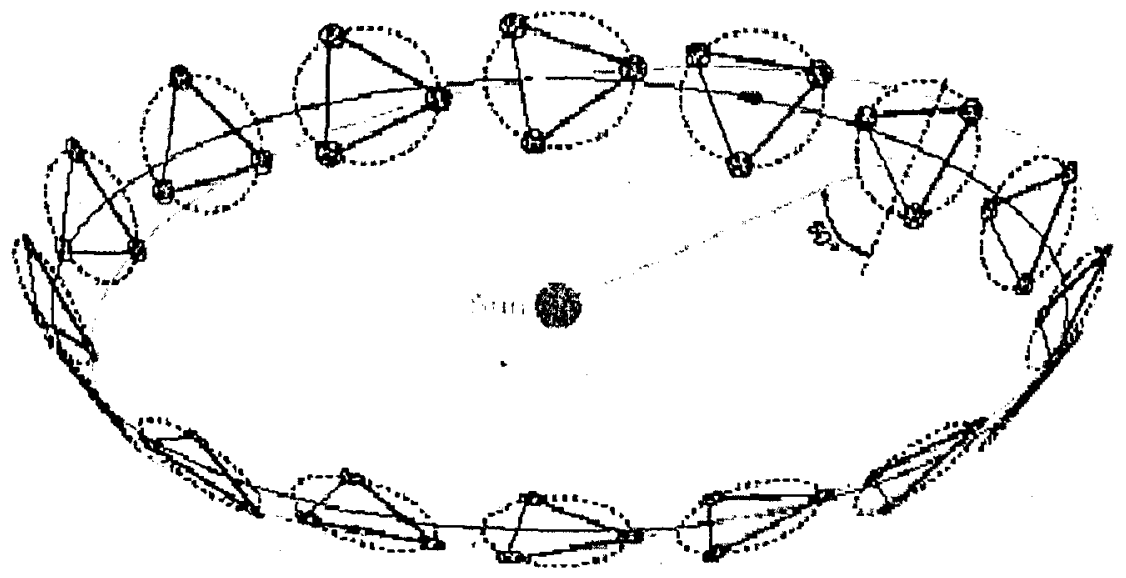

Fig. 5: Spacecraft orbits about the sun 


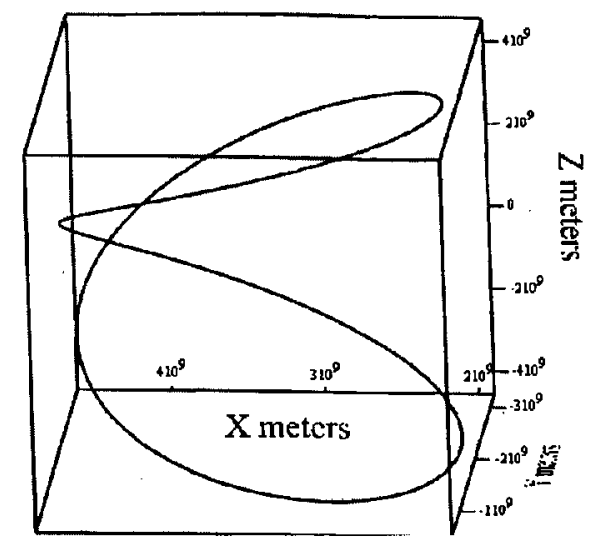

Fig. 6: Motion of distant $\mathrm{SC}$ with respect to a locally inertial frame.

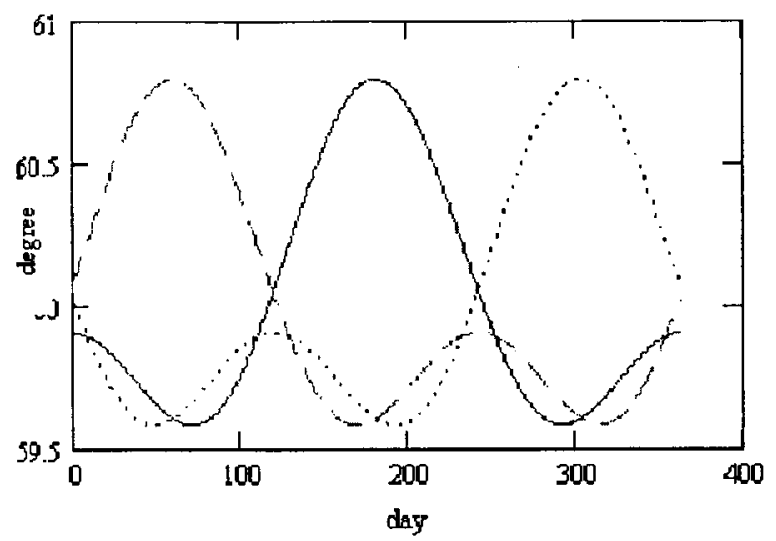

Fig. 7: Angular separation of distant SC in course of a year.

The beam's transit time between $\mathrm{SC}$ is about $16.7 \mathrm{sec}$. To maximize the received signal the outgoing beam is aimed slightly ahead of the distant SC. This means that the sending and receiving directions are slightly different and in the course of a year as shown in fig. 8. One more motion (excluding SC jitter) should be mentioned as it to has a slight effect on polarization. As can be seen in Fig. 5 the plane containing the three SC rotates in a year. In a sense this is the plane of "incidence" of the three SC. This rotation is not constant. Fig.9 shows the magnitude and variation of

$$
\frac{\cos ^{-1}(\widehat{N}(t+16.67) \cdot \widehat{N}(t))}{16.67 \mathrm{sec}}
$$

where $\widehat{N}(t)$ is the unit normal to the plane defined by the three SC at time $t$. Keplerian orbits were used in the creation of Fig. 6-9.

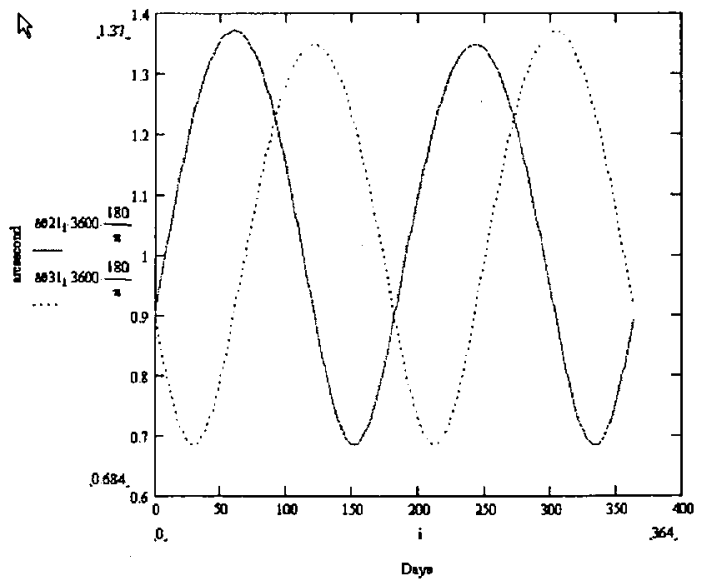

Fig. 8: Point ahead variation in the course of a year.

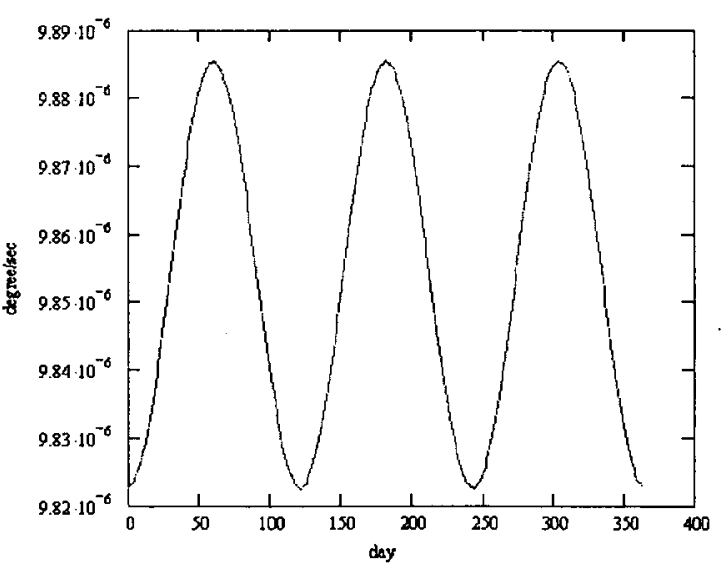

Fig. 9: Rotation of the SC plane. 


\section{Far Field}

The far field beam intensity is determined by evaluating (13) for each polarization component separately.

$$
E(X, Y, Z)=\iint_{\text {drea }} \frac{E(x, y, z) \cdot e^{i \frac{2 \pi R}{\lambda}\left(\omega_{x} x+\omega_{y} y\right)} e^{\left(\frac{x^{2}+y^{2}}{\text { Waist }}\right)}}{\text { Dist }} d x d y
$$

The integral (13) is over the exit aperture of a telescope and Dist is the distance between SC. A one watt Gaussian beam (clipped by the aperture, with no spiders holding the secondary and no wave front aberrations) produces the far field intensity pattern shown in fig. 10a-b. For maximum signal the distant SC is at the intensity peak where the intensity is a few tens of picowatts/meter ${ }^{2}$.

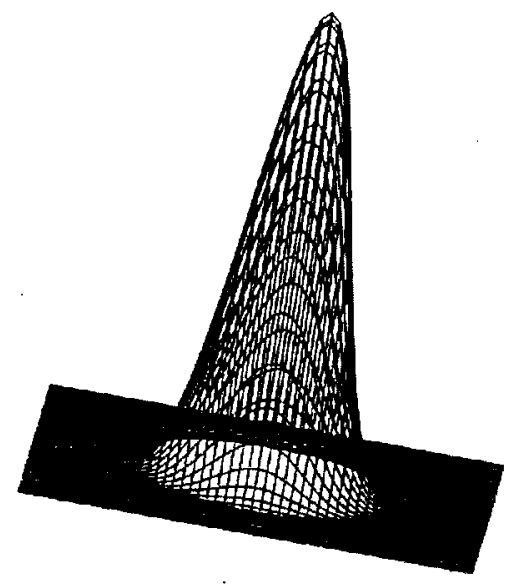

Fig. 10a: Far field intensity

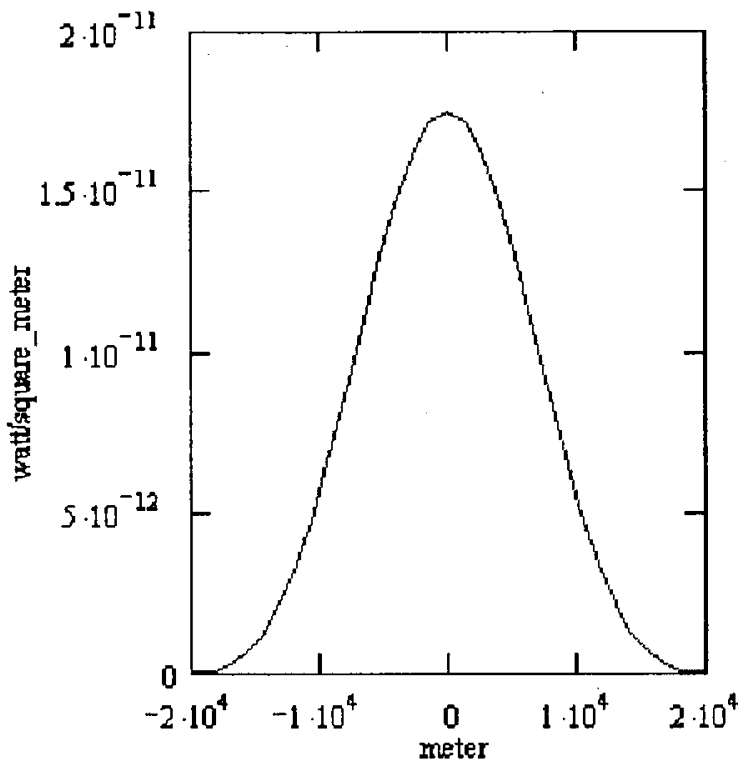

Fig. 10b: Far field intensity cross-sectional slice.

\section{Entrance Aperture to Proof Mass to Detector - Incoming Beam}

Once we have computed the electric field at approximately 16.7 second future position of the distant SC we use it as the starting point for a conventional ray trace. At the telescope aperture we have a circularly polarized $E$ field. If the telescope is mis-aligned (possibly caused by SC jitter) with the incoming beam there will be some tilt. The rays acquire the intensity and phase at their starting point and propagate through the telescope, through the quarter wave plate which now converts the light into a linear polarization such that it passes through the polarizing beam splitter, in fig. 1-2, then through another quarter wave plate to be converted to circular polarization, then reflection from the proof mass where the handedness' of the circular polarization changes and consequently, when converted by the polarizing beam splitter to linear polarized light again is now reflected by the beam splitter to the main quadrant photodiode. The use of Jones matrices and diagrams helps in following this argument.

\section{Single Ray - Beginning to End}

Here we illustrate the statements in the previous section with diagrams accompanied by Jones matrices. A ray which starts at the fiber, fig. 1-2, becomes strongly linearly " $S$ " polarized on passing through the birefringent prisms and is then incident on 
the polarizing beam splitter which reflects " $S$ " polarized light. To simplify the discussion the ray will be in the plane of the paper. The " $S$ " polarization state is perpendicular to the page and " $P$ " is in the page. The Jones matrices for a quarter wave plate $Q W$, a reflection refl and rotation Rot are given by (14-16).

$$
\begin{gathered}
Q W=\left(\begin{array}{cc}
1 & 0 \\
0 & e^{-i \frac{\pi}{4}}
\end{array}\right) \\
r e f l=\left(\begin{array}{cc}
1 & 0 \\
0 & -1
\end{array}\right) \\
\operatorname{Rot}(\theta)=\left(\begin{array}{cc}
\cos \theta & \sin \theta \\
-\sin \theta & \cos \theta
\end{array}\right)
\end{gathered}
$$

In fig. 11 , we start with a ray having only a "S" component $\vec{E}_{1}$ which is reflected by the polarizing beam splitter. After going through the quarter wave plate we get, fig. 2 , circularly polarized light $\vec{E}_{2}$. The two reflections from the secondary and primary give a unit matrix and are ignored as are ordinary refractive lenses. The ray now encounters, having traveled from telescope to telescope, another quarter wave plate which gives us the "P" polarized $\vec{E}_{3}$ and this polarization is transmitted through the beam splitter. The ray now passes through another quarter wave plate giving circular polarized $\vec{E}_{4}$, which upon reflection by the proof mass changes handedness to $\vec{E}_{5}$ and after the quarter wave plate becomes linearly " $\mathrm{S}$ " polarized $\vec{E}_{6}$ which is reflected by the " $S$ " reflecting beam splitter toward the main quadrant photodiode.
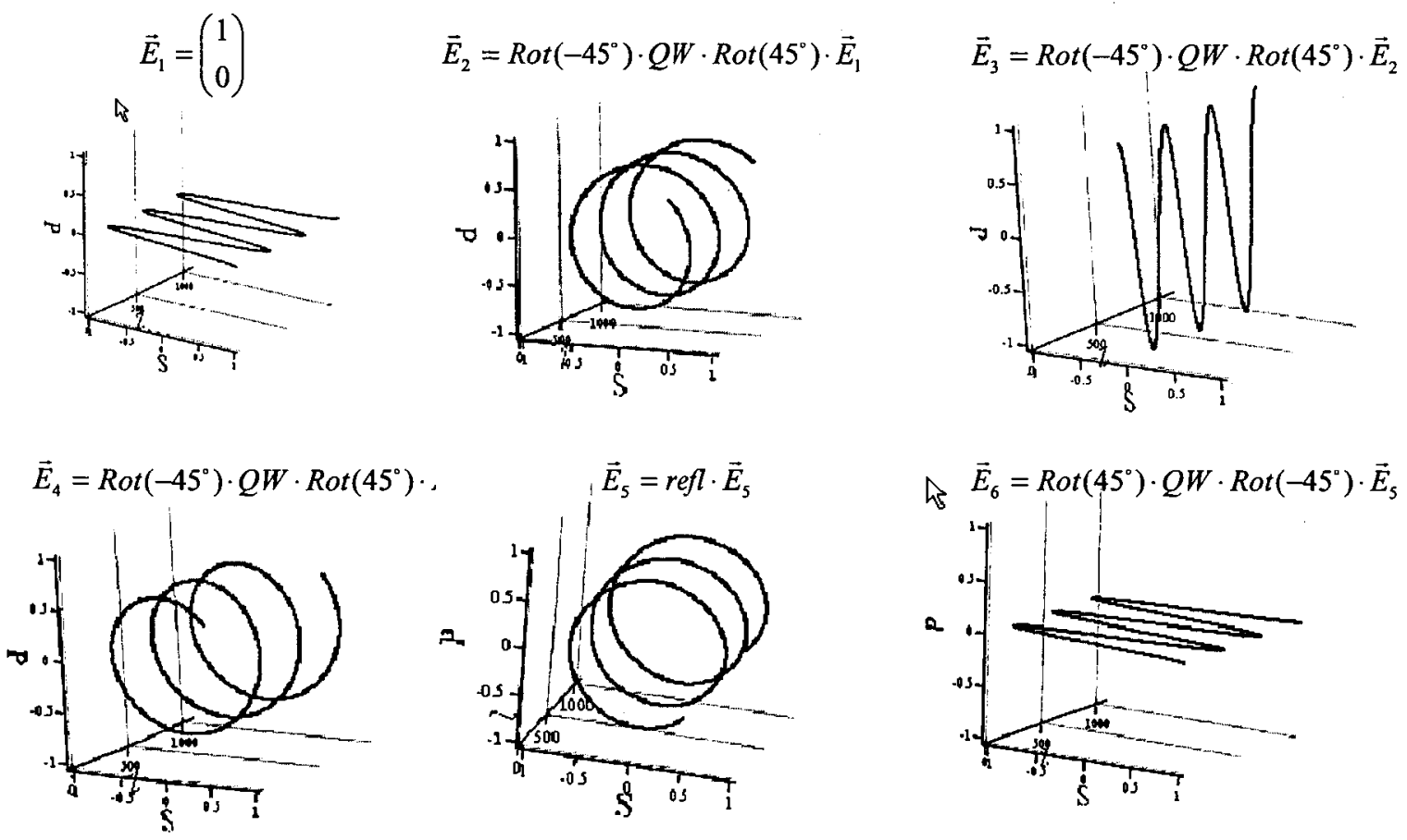

Fig. 11: Polarization States after various elements.

It should be noted that the sequence of polarization state changes within the LISA optical bench and between the SC is not final. Linearly polarized light between the $\mathrm{SC}$ is being considered. The decision will be based upon a careful examination of 
the available transmissive and thin film coating materials, there stability with temperature and time and the results of this and other PRT analyses.

\section{Conclusion}

An outline of the end-to-end polarization ray trace analysis has been presented. A preliminary optical design of both the receive and transmit optics have been created and used in the PRT. The polarization switch has been "turned on" in the commercial codes resulting in default coatings being applied to transmissive optical elements with the reflectors treated as either ideal of bare metal. Real coatings on the optical elements have not been designed, but they should be very simple as they need only enhance reflection or transmission of monochromatic light. The polarizing beam splitter will probably have the most complicated coating. The next enhancement to this PRT model will be to use text book coatings in RAYPKS and the commercial codes and compare results. A bit later as the optical design matures there will be discussions with coating vendors regarding real coatings their manufacturability to LISA's requirements and to incorporated actual performance into the model. Of course it is hoped that the final result of the PRT modeling effort is a computational procedure which improves our ability to predict the noise in LISA's optical path from laser to detector.

\section{Acknowledgments}

As with any modeling effort, the authors wish to thank co-workers located at Goddard, JPL and ESA for their suggestions and work. Goddard's optics group, Mark Wilson in particular, provided a good deal of motivation to use the in-house RAYPKS PRT code so that a comparison of its results could be made with the results of commercial codes, thus giving insight into both. Of course thanks go to NASA for the funding which made this work possible.

\section{References}

1. Laser Interferometer Space Antenna for the detection and observation of gravitational waves, Pre-Phase A Report, Second Edition, July 1998

2. LISA Final Technical Report, astrium report \# LI-RP-DS-009, April 2000

3. C. Harb, A. Hirai, "Interim Technical Report: Polarization Measurements", JPL

4. E. Waluschka, E., "LISA Optics Model”, Proceedings SPIE, Vol. 4852, (July, 2002)

5. Waluschka,E., "Polarization Ray Trace",Opt. Eng.,Vol 28,Number 2, 86-89 (1989) 